\title{
Radiation-induced lung damage in patients treated with stereotactic body radiotherapy after EGFR-TKIs: is there any difference from stereotactic body radiotherapy alone?
}

\author{
Xingni Tang ${ }^{1}$, Yichao Shen ${ }^{1}$, Yinnan Meng ${ }^{1}$, Liqiao Hou ${ }^{1}$, Chao Zhou ${ }^{1}$, Changhui Yu ${ }^{1}$, Haijian Jia ${ }^{1}$, \\ Wei Wang', Ge Ren ${ }^{2}$, Jing Cai ${ }^{2}$, X. Allen $\mathrm{Li}^{3}$, Haihua Yang ${ }^{1 \#}$, Feng-Ming (Spring) Kong ${ }^{1,4,5,6 \#}$ \\ ${ }^{1}$ Laboratory of Cellular and Molecular Radiation Oncology, Radiation Oncology Institute of Enze Medical Health Academy, Department of \\ Radiation Oncology, Taizhou Hospital Affiliated to Wenzhou Medical University, Taizhou, China; ${ }^{2}$ Department of Health Technology and \\ Informatics, The Hong Kong Polytechnic University, Hung Hom, Hong Kong, China; ${ }^{3}$ Department of Radiation Oncology, Medical College of \\ Wisconsin, Milwaukee, WI, USA; ${ }^{4}$ Department of Clinical Oncology, Hong Kong University Shenzhen Hospital, Shenzhen, China; ${ }^{5}$ Hong Kong \\ University LKS Medical School, Hong Kong, China; ${ }^{6}$ Department of Radiation Oncology, University Hospitals Cleveland Medical Center, Case \\ Western University, Case Comprehensive Cancer Center, Cleveland, OH, USA \\ Contributions: (I) Conception and design: H Yang, FM Kong; (II) Administrative support: H Yang, W Wang, XA Li, J Cai, FM Kong; (III) Provision \\ of study materials or patients: Y Meng, C Zhou, W Wang; (IV) Collection and assembly of data: X Tang, Y Shen, L Hou, C Yu, H Jia, G Ren; (V) \\ Data analysis and interpretation: X Tang, Y Shen, G Ren, L Hou, H Jia; (VI) Manuscript writing: All authors; (VII) Final approval of manuscript: All \\ authors. \\ \#These authors contributed equally to this article. \\ Correspondence to: Haihua Yang, MD. Laboratory of Cellular and Molecular Radiation Oncology, Radiation Oncology Institute of Enze Medical \\ Health Academy, Department of Radiation Oncology, Taizhou Hospital affiliated to Wenzhou Medical University, Taizhou 317000 , China. \\ Email: yhh93181@hotmail.com; Feng-Ming (Spring) Kong, MD, PhD. Laboratory of Cellular and Molecular Radiation Oncology, Radiation \\ Oncology Institute of Enze Medical Health Academy, Department of Radiation Oncology, Taizhou Hospital Affiliated to Wenzhou Medical \\ University, Taizhou 317000, China; Department of Clinical Oncology, Hong Kong University Shenzhen Hospital, Shenzhen, China. \\ Email: springenzert@163.com; kong0001@hku.hk.
}

Background: To quantitatively evaluate lung damage after treatment of epidermal growth factor receptor tyrosine kinase inhibitors (EGFR-TKIs) and stereotactic body radiotherapy (SBRT) in patients with nonsmall cell lung cancer (NSCLC), and compare that of SBRT only treatment.

Methods: Eligible patients from an IRB-approved prospective clinical trial had one month of EGFRTKIs treatment followed by SBRT (TKI + SBRT) and with 3-month follow-up high resolution CT. NSCLC patients treated with SBRT alone during the same time period without EGFR-TKIs or other systemic therapies were identified as controls. The lung damage was assessed clinically by pneumonitis and quantitatively using by CT intensity (Hounsfield unit, HU) changes. The mean HU values were extracted for regions of the lungs receiving the same dose range at $10 \mathrm{~Gy}$ intervals to generate dose-response curves (DRC). The relationship of HU changes and radiation dose was modeled using a Probit model.

Results: Four out of 20 (25\%) TKI + SBRT patients and none of 19 (0\%) SBRT alone patients had developed grade 2 and above pneumonitis $(\mathrm{P}=0.053)$, respectively. Sixty percent of TKI + SBRT patients and $30 \%$ SBRT alone patients had HU changes of the normal lung density $>200 \mathrm{HU}$, respectively. There were significant differences in the DRC and in lung HU changes between the two groups (all $\mathrm{P}<0.05)$. The physical dose for a $50 \%$ complication risk $\left(T D_{50}\right)$ of CT lung damage was 52 Gy (CI: 46-59) in TKI + SBRT group versus 72 Gy (CI: 58-107) in SBRT alone group $(\mathrm{P}<0.01)$.

Conclusions: Compared to patients treated with SBRT alone, patients treated with EGFR-TKIs followed by SBRT were more incline to develop radiation pneumonitis, and resulted in greater lung CT intensity changes and steeper dose-CT lung damage response relationship at 3 months post treatment. Future study with larger number of patients and longer follow-up period is warranted to validate this finding. 
Keywords: Epidermal growth factor receptor tyrosine kinase inhibitor (EGFR-TKIs); stereotactic body radiotherapy (SBRT); quantitative CT analysis; normal tissue complication probability (NTCP); lung damage

Submitted May 14, 2020. Accepted for publication Dec 17, 2020.

doi: 10.21037/apm-20-1116

View this article at: http://dx.doi.org/10.21037/apm-20-1116

\section{Introduction}

Epidermal growth factor receptor tyrosine kinase inhibitors (EGFR-TKIs) have been widely used in cancer treatment. Multidisciplinary international practice guidelines (NCCN, IASLC) recommend a combination of stereotactic body radiotherapy (SBRT) with EGFR-TKIs to improve treatment efficacy for advanced non-small cell lung cancer (NSCLC) (1). The outcome of the combined therapy in unresectable patients with advanced NSCLC is encouraging (2-4). A recent study showed that the patients treated with SBRT and EGFR-TKIs had a significantly improved overall survival at one year as compared to the conventional radiation therapy (RT) (71.5\% vs. 36.5\%) (5). Laboratory and clinical studies suggested that EGFR-TKIs can enhance the radiosensitivity of tumor cells by increased killing of the remaining radiation resistant cells $(6,7)$.

Although the volume of adjacent normal lung tissue receiving high dose is limited, SBRT lung toxicity is a clinically significant issue, and its association with the combined EGFR-TKIs treatment is unknown. To better understand whether EGFR-TKIs alter the radiosensitivity of normal lung cells, this study aimed to investigate early lung injury in patients treated with SBRT following EGFRTKIs, comparing to those treated with SBRT alone, using quantitative CT imaging analysis of the lung density acquired before and after the treatments. We present the following article in accordance with the STROBE reporting checklist (available at http://dx.doi.org/10.21037/apm-20-1116).

\section{Methods}

\section{Study population and treatment details}

The primary study population consisted of 20 consecutive NSCLC patients (from 2016 to 2018) treated with EGFRTKIs (125 mg Icotinib, three times daily) for a month followed by SBRT in an Institutional Ethic Committee approved prospective study (8). For comparison, NSCLC patients treated with similar SBRT technique during the same time period without TKI or any other systemic therapy were identified as controls. To retrieve enough number of patients for the control group, we had extended the patient treatment record to 2013. Patients with previous RT or without 3 months follow-up CT scans were excluded. Patients were assessed blindly and graded for tumor response and radiation pneumonitis by two senior radiation oncologists according to a consensus report by Kong et al. with consideration of CTCAE for grading $(9,10)$. The study was conducted in accordance with the Declaration of Helsinki (as revised in 2013). The study was approved by the Taizhou Hospital Ethical Committee (No. KB20170501) and informed consent was taken from all the patients.

The prescription dose was 50 Gy in 5 fractions for peripheral lesions or $60 \mathrm{~Gy}$ in 8 fractions for central lesions, which covered more than $95 \%$ of the planning target volume (PTV) except when tumor was adjacent to the critical structure. All patients were positioned head-firstsupine and were immobilized with thermoplastic mask. CT scans of free breathing, unenhanced and enhanced, and four-dimensional (4DCT) were acquired using a CT scanner (discovery CT590 RT, GE) during the simulation. Unenhanced free breathing CTs were obtained using the same scanner 3 months after SBRT. The gross tumor volumes (GTV) and organs at risk (OARs) including normal lung tissue were contoured by the treating radiation oncologists on the unenhanced free breathing CT sets. The PTV was generated by adding a margin to the GTV according to the Radiation Therapy Oncology Group Phase II clinical trial on hypo fractionated stereotactic lung radiation therapy (RTOG 0236). Each SBRT plan was delivered with 9- to 11-field intensity-modulated radiotherapy (IMRT). The dose calculation was done with collapsed cone convolution algorithm (CCC) (Pinnacle, Philips Medical Systems, Milpitas, CA). The pre-SBRT planning CT and post-SBRT CT were rigidly registered based on both bony anatomy and tumor structure. (Eclipse, Varian Medical Systems, Palo Alto, CA). The quality of registration was confirmed by at least one medical physicist and one radiation oncologist independently. Isodose lines of $10,20,30,40,50$, and 60 Gy were converted into 
contours by excluding the PTV on the planning CT and populated onto the corresponding post-SBRT CT. Clinical radiation lung damage was assessed and graded per treating physicians, according to CTCAE4.0.

\section{HU measurements and dose-response curves}

The mean CT number, i.e., Hounsfield number (HU) of an exclusive region was calculated by Boolean calculation of masks derived from the isodose line contours using MATLAB (Math Works, Natick, MA) following a method described previously (11). For example, the mean HU of the exclusive region between the isodose lines of $10 \mathrm{~Gy}$ and 20 Gy in lung can be calculated using the following formula (11): $\left[M_{10 G y}\right.$ isodose line AND (NOT $M_{20 G y}$ isodose line) $]$ AND ( $M_{\text {Lung-R }}$ OR $\left.M_{\text {Lung-L }}\right)$, where AND, NOT, and OR are Boolean operators, and $M_{10 \text { Gy isodose line, }} M_{20 \text { Gy isodose line, }} M_{\text {Lung-R }}$ and $M_{\text {Lung-L }}$ are the masks with their self-revealing subscripts. The change of the mean HUs $(\Delta \mathrm{HU})$ within each region of lung receiving the same dose $(d)$ between the pre- and post-SBRT CTs were calculated as follows: $\Delta \mathrm{HU}(\mathrm{d})=\mathrm{HU}_{\text {post-SBRT }}(d)-$ $\mathrm{HU}_{\text {pre-SBRT }}(d)$. The dose-CT number association curves of preand post-SBRT datasets were then fitted to a linear-quadratic function. For each group, dose-response curves (DRC) for normal lung tissue were achieved by fitting a linear-quadratic function between $\Delta \mathrm{HU}$ and the corresponding dose bins. In order to normalize $X$ axis, the dose bins were converted to biologically effective dose (BED) with an $\alpha / \beta$ ratio of 3.6 Gy (Eq.[1]) (12). To simplify the analysis, two different dose prescriptions were unified using BED according to the formula (Eq. [2]).

$$
\begin{aligned}
& B E D=n d\left(1+\frac{d}{\alpha / \beta}\right) \\
& n d=n\left(-\frac{\alpha}{2 \beta}+\sqrt{\frac{\alpha}{\beta} \frac{B E D}{n}+\left(\frac{\alpha}{2 \beta}\right)^{2}}\right)
\end{aligned}
$$

\section{Normal tissue complication probability (NTCP)}

The Lyman-Kutcher-Burman (LKB) NTCP model was established for CT lung damage by using a dichotomous toxicity endpoint for lung density increase. CT lung damage was defined by the thresholding the HU change of $200 \mathrm{HU}$ for the lung NTCP computation (13). Assuming the dose for each bin was relatively uniform, the model can be simplified to

$$
N T C P(D)=\frac{1}{\sqrt{2 \pi}} \int_{-8}^{t} e^{-\frac{u^{2}}{2}} d u
$$

$$
\text { With } t=\frac{D-T D_{50}}{m \cdot T D_{50}}
$$

where $\mathrm{D}$ is equivalent uniform dose (EUD), $T D_{50}$ is the dose that results in $50 \%$ complication risk for a given partial volume and $m$ is a slope parameter for the sigmoid curve. To determine $T D_{50}$ and $\mathrm{m}$ values, a Probit model was fitted to the patient data using the following maximum loglikelihood function:

$$
L L=-\sum_{i} y_{i} \log \left(\operatorname{NTCP}\left(D_{i}\right)\right)=\left(1-y_{i}\right) \log \left(1-\operatorname{NTCP}\left(D_{i}\right)\right)
$$

which is described for all data points $i$ with the binary variable $y i$ being 1 for $\Delta \mathrm{HU} \geq 200 \mathrm{HU}$ and 0 for $\Delta \mathrm{HU}<200 \mathrm{HU}$. Di is referred to a delivered mean dose for data point $i$; NTCP(Di) is the probability calculated by Eq. [3] for $\Delta \mathrm{HU}$ $\geq 200 \mathrm{HU}$ at point $i$. The $95 \%$ confidence intervals (CI) for parameter $T D_{50}$ and $\mathrm{m}$ were obtained by profile likelihood method (14). Briefly, the lower and upper bounds of CI were obtained from two values which satisfy the equality with the optimal likelihood reduced by $x^{2}(0.05,1) / 2=1.92$.

\section{Lung function assessment}

To test comparability of the patients local lung function before SBRT, lung functional maps were generated using a CT based lung functional mapping method (15), which included image preprocessing and residual neural network computation. The 4D CT images of various breathing phases were first co-registered, resampled, and resized. The contrast of the masked CT was adjusted using the histogram equalization to allow the low intensity region to gain a higher contrast. Then a histogram-based regularization approach was used to normalize the values on the functional map. The segmented images were further cropped and resized to reduce the consumption of the computation. A 3D neural network model was built to learn a direct mapping from the processed CT to ventilation images. The model was previously trained and tested using CT scans of 41 patients. Binary cross entropy with auxiliary correlation was used as the loss function.

\section{Statistical analysis}

The primary endpoint of this study was radiation lung 
damage which included clinical pneumonitis and CT lung damage defined by CT intensity. Statistical analyses were performed using SPSS statistical software (version 17.0, IBM, Armonk, NY) with P values $<0.05$ considered statistically significant. Chi-square test was used for qualitative analyses, while student $t$-test was used for quantitative data. The correlation between the $\Delta \mathrm{HU}$ value and the regional dose was analyzed using a quadratic model fitting. The estimated correlation coefficient $\left(\mathrm{R}^{2}\right)$ was used to compare the quality of analyses in two groups. The differences between the fit parameters from SBRT alone and target SBRT (SBRT plus EGFR-TKI) groups were evaluated using the likelihood ratio test (16).

\section{Results}

\section{Characteristics of the study population}

Twenty patients enrolled in the prospective study of one-month EGFR-TKI treatment followed by SBRT treatment, forming the primary study population, the TKISBRT group. The control group included 19 patients (20 lesions), with 1 patient treated with SBRT for two different locations in two different time courses (3 months follow-up CTs available for each course). The control patients were identified from a database of 242 NSCLC patients treated with SBRT from September 2013 to July 2018. Detailed characteristics of the two groups of patients are shown in Table 1. There were no significant differences in age, performance status, tumor size, tumor location and SBRT dose prescription between the two groups. There was a significant difference in gender (more female in TKI + SBRT group), smoking history (more smokers in SBRT alone group), histology (more squamous cell carcinoma in SBRT alone group), and lung volume (greater lung volume in SBRT alone group) (all $\mathrm{P}<0.05)$ between the two groups. There were no significant differences in tumor response between the two groups (Table 2).

\section{Radiation lung damage}

Four of 20 patients (25\%) treated with TKI + SBRT developed clinical lung damage, i.e., grade 2 and above radiation pneumonitis comparing to 0 of 20 patients $(0 \%)$ in the SBRT alone group (Table 2, $\mathrm{P}=0.053$ ). There were significant differences in changes of CT intensity at 3 months post SBRT from that of pre-SBRT CTs. Patient 1 and 2 displayed in Figure 1 were two representative examples from SBRT alone group and TKI + SBRT group respectively. They were matched in gender, smoking status, tumor primary size and location, dose prescription and lung volume. Figure $1 A, B, C$ for patient 1 showed a pre-treatment lung ventilation map, pre- and post-treatment CT scans with SBRT dose distributions, respectively. Figure 1D,E,F was the corresponding examples for patient 2 . As shown in Figure 1, no remarkable differences between two patients were observed in lung ventilation and CT scans before treatment. However, patient 2 got more significant $\mathrm{HU}$ changes in CT scans at 3 months after the end of treatment.

Figure $2 A$ shows the dose- $\Delta H U$ relationships of the two groups. The data were fitted well to a linear-quadratic function $\left(R^{2}>0.5\right)$. The maximum difference of $\Delta H U$ value between the two groups got at the BED dose of $168 \mathrm{~Gy}$. Compared to SBRT alone group, TKI + SBRT group shows a greater increase in $\mathrm{HU}$ values. The thresholding BEDs for any increase in HU was the 27 Gy for TKI + SBRT group versus 46 Gy in SBRT alone. At the dose of 189 Gy BED (physical dose 50 Gy in 5 fractions), there was a difference of $123 \mathrm{HU}$ in $\Delta \mathrm{HU}$ value between TKI-SBRT and SBRT groups $(\mathrm{P}=0.034)$.

\section{Lung NTCP approximation}

The comparison between the lung NTCP models of the TKI + SBRT group and the SBRT alone group is shown in Figure $2 B$. BED was used to compute NTCP of CT lung damage, defined by the HU increase $\geq 200 \mathrm{HU}$. The maximum likelihood Probit fitted parameters $T D_{50}$ (dose resulting in $50 \%$ complication risk) was 72 Gy physical dose (CI: 58-107 Gy) in SBRT alone group versus 52 Gy (CI: 46-59 Gy) in SBRT plus EGFR TKI group. The $\mathrm{m}$ values (slope of the sigmoid curve) were 0.48 (CI: 0.38-0.62) for EGFR TKI plus SBRT versus 0.49 (CI: 0.38-0.66) for the SBRT alone. The difference of $T D_{50}$ between the groups was statistically significant $(\mathrm{P}<0.001)$. For practical convenience, the physical doses resulting in different NTCP values of each group are listed in Table 3.

\section{Matched comparisons}

Considering that the two groups of patients were not matched in patient characteristics likely associated with radiation-induced lung damage (such as gender, lung volume and smoking status), additional efforts have been taken to identify cases matched for baseline lung function, which is one of the most important factors for radiation 
Table 1 Patient and treatment characteristics

\begin{tabular}{|c|c|c|c|c|c|}
\hline Characteristic & \multicolumn{2}{|c|}{ SBRT alone $(\mathrm{N}=20)$} & \multicolumn{2}{|c|}{ TKI + SBRT $(\mathrm{N}=20)$} & $P$ value \\
\hline Age (years) & 70 & $50-83$ & 68 & $62-80$ & 0.179 \\
\hline Sex & & & & & 0.048 \\
\hline Male & 16 & $80 \%$ & 9 & $45 \%$ & \\
\hline Smoking history & & & & & 0.01 \\
\hline Smoker & 15 & $75 \%$ & 6 & $30 \%$ & \\
\hline Non-smoker & 5 & $25 \%$ & 14 & $70 \%$ & \\
\hline KPS score & & & & & 0.254 \\
\hline Histology & & & & & 0.008 \\
\hline Adenocarcinoma & 11 & $55 \%$ & 19 & $95 \%$ & \\
\hline Squamous cell carcinoma & 9 & $45 \%$ & 1 & $5 \%$ & \\
\hline Stage & & & & & $<0.001$ \\
\hline $\mathrm{I}(\mathrm{a} / \mathrm{b})$ & 16 & $80 \%$ & 0 & $0 \%$ & \\
\hline II (a/b) & 4 & $20 \%$ & 0 & $0 \%$ & \\
\hline III (a/b) & 0 & $0 \%$ & 4 & $20 \%$ & \\
\hline IV & 0 & $0 \%$ & 16 & $80 \%$ & \\
\hline \multicolumn{6}{|l|}{ GTV } \\
\hline Diameter $(\mathrm{cm})$ & 3.2 & $1.4-5.4$ & 3.0 & $1.6-5.1$ & 0.502 \\
\hline Volume $\left(\mathrm{cm}^{3}\right)$ & 23.9 & $1.3-81.2$ & 17.9 & $2.1-70.9$ & 0.363 \\
\hline Lung volume $\left(\mathrm{cm}^{3}\right)$ & $4,083.6$ & $1,749.2-7,425.2$ & $3,046.3$ & $1,762.3-4,981.5$ & 0.011 \\
\hline RT fractions $(n) /$ dose per fraction & & & & & 0.48 \\
\hline $5 \times 10 \mathrm{~Gy}$ & 13 & $65 \%$ & 16 & $80 \%$ & \\
\hline $8 \times 7.5$ Gy & 7 & $35 \%$ & 4 & $20 \%$ & \\
\hline
\end{tabular}

GTV, gross tumor volume; KPS, Karnofsky performance status; RT, radiotherapy; SBRT, stereotactic body radiation therapy. 
Table 2 Patients' response evaluation and pneumonitis grading

\begin{tabular}{|c|c|c|c|}
\hline Characteristic & SBRT alone $(\mathrm{N}=20)$ (No. of lesions) & Targeted SBRT (N=20) (No. of lesions) & $P$ value \\
\hline PR & 19 & 18 & \\
\hline SD & 1 & 2 & \\
\hline Pneumonitis grading & & & 0.053 \\
\hline Grade 2 & 0 & 4 & \\
\hline
\end{tabular}

$\mathrm{PR}$, partial response; SD, stable disease.
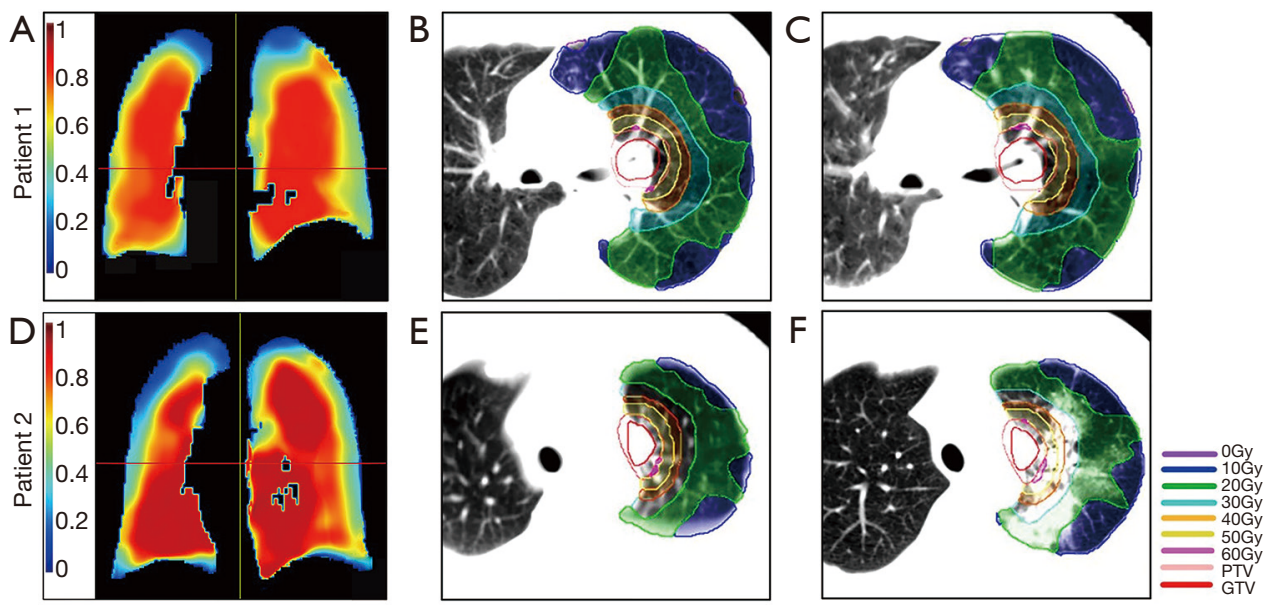

Figure 1 Lung ventilation of representative patients and CT changes after SBRT with and without EGFR-TKI treatment. Representative examples of the comparison of CT changes at three months after treatment. Both patients were treated with the same prescription dose prescription of 60 Gy in 8 fractions and had similar clinical characteristics, including age and gender. A pre-treatment lung ventilation map (A), a pre- and 3 months post treatment CT scan with SBRT dose distribution (B and C) for the patient 1 treated with SBRT alone; a pretreatment lung ventilation map (D), a pre- and 3 months post treatment CT scan with SBRT dose distribution (E and F) for the patient 2 treated with EGFR-TKI plus SBRT. Isodose lines are shown from purple (0 Gy) to pink (prescription dose). Gross tumor volume (GTV) is shown in red range. SBRT, stereotactic body radiation; EGFR-TKI, epithelial growth factor receptor tyrosine kinase inhibitor.

lung damage. Six pairs were identified by matching gender, smoking status, tumor primary size and location dose prescription and lung volume (Table 4). Thirty-nine patients with 4D-CT of the whole lung were identified to generate ventilation maps (other patients only had 4D CT covering the tumor regions). The details of the lung function assessment comparing matched pairs of patients was shown in Table 4. There were no significant differences between two groups $(\mathrm{P}>0.05)$. Figure $1 A$ and $1 D$ shows the ventilation maps of two representative cases. Overall, the SBRT alone patients seemed to have modestly lower functional activity, yet there was no remarkable defects or differences around the radiating fields on visual inspection.

\section{Discussion}

To our knowledge, this is the first study assessing SBRT associated lung damage using quantitative lung CT intensity changes for primary NSCLC patients treated TKI + SBRT, compared with SBRT alone. Significant more increase in lung HU changes after SBRT was observed in the TKI + SBRT group as compared to the SBRT alone group. The NTCP modeling indicated that the dose generating the same level of lung damage NTCP was about $40 \%$ lower in 
A

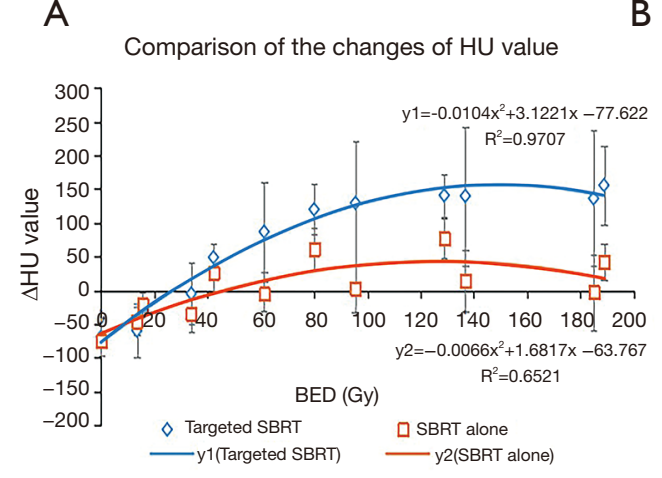

B

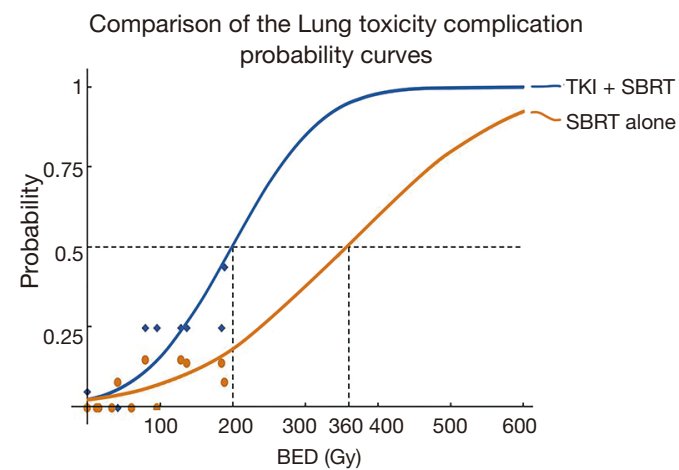

Figure 2 CT intensity dose response relationships and lung toxicity complication probability curves of SBRT with and without EGFRTKI treatment. CT scans were all performed around 3 months after completion of SBRT. (A) The dose-response curve between SBRT alone (square point) and TKI + SBRT groups (diamond point). The data was fitted to a linear-quadratic function with dose bin converted to biologically effective dose (BED). Error bars represent $95 \%$ confidence intervals. The differences between SBRT alone group and Target SBRT group shown in Figure 2 make a statistic significance $(\mathrm{P}<0.05)$. (B) The lung toxicity complication probability curves of SBRT with and without EGFR-TKI treatment. Probability of CT lung damage (defined as a density increase of $200 \mathrm{HU}$ ) with biologically effective dose for SBRT with prior EGFR-TKI group (diamond points and solid line) and SBRT alone group (dot points and dotted line). The model parameter $T D_{50}$ (dose resulting in 50\% complication risk) reported as physical dose was 52 Gy (95\% CI: 46 -59 Gy) in EGFR TKI + SBRT group versus 72 Gy (95\% CI: 58-107 Gy) in SBRT alone group, with the corresponding $m$ values (slope of the sigmoid curve) of 0.48 (CI: 0.38-0.62) versus 0.49 (CI: 0.38-0.66). SBRT, stereotactic body radiation; EGFR-TKI, epithelial growth factor receptor tyrosine kinase inhibitor.

Table 3 Dose comparison for two groups resulting different normal tissue complication probability

\begin{tabular}{lccc}
\hline Parameter & SBRT alone $(n=5)(G y)$ & TKI + SBRT $(n=5)(G y)$ & Diff in Gy (\%) \\
\hline TD $_{10}$ & 42 & 29 & 46.3 \\
TD $_{20}$ & 54 & 38 & 42.2 \\
TD $_{30}$ & 61 & 43 & 41.0 \\
TD $_{40}$ & 67 & 48 & 40.1 \\
TD $_{50}$ & 72 & 52 & 39.7 \\
\hline
\end{tabular}

TDx: dose resulting $\mathrm{x} \%$ complication risk when $\mathrm{n}=5$; diff: the percentage of dose difference in two groups in reference to SBRT alone group. SBRT, stereotactic body radiation therapy; TKI, tyrosine kinase inhibitor.

the TKI + SBRT group than that of the SBRT alone group.

Although the combination of radiation with EGFRTKIs is considered to be a safe and effective strategy to treat patients with advanced NSCLC, results from our study suggest an increased risk of lung toxicity after the combined modality treatment, which is clinically significant. Our finding of $40 \%$ lower $\mathrm{TD}_{50}$ is consistent with a previous study, in which the radiation effect of the adding gefitinib to the single-dose radiation was found to be equivalent to using a $60 \%$ higher radiation dose (17). While external validation study is needed, the results from this study also suggest that
EGFR TKI may sensitize the radiation injury of lung tissue that may be quantitatively measured using CT.

Our finding of TKI + SBRT increasing sensitivity of radiation lung damage has clinical significance. Both EGFRTKIs and radiation carry potential risk of lung injury which may affect the patient's quality of life. The reported rates of grade 3 and above pneumonitis ranged 3\% to $20 \%$ (18-21). Gefitinib and erlotinib are two types of EGFRTKIs widely used in the clinic. It has been reported from a phase I study that grade 3 and 4 pulmonary toxicity was occurred in $20 \%$ of patients with stage III NSCLC treated 
Table 4 The information of six maximum matched pairs of patients

\begin{tabular}{|c|c|c|c|c|c|c|c|c|c|c|c|c|c|}
\hline \multirow{2}{*}{ No. } & \multirow{2}{*}{ Sex } & \multirow{2}{*}{ Dose prescription } & \multirow{2}{*}{$\begin{array}{l}\text { Tumor } \\
\text { position } \\
\text { in lung }\end{array}$} & \multicolumn{2}{|c|}{ Age (group $A / B$ ) } & \multicolumn{2}{|c|}{ Lung volume $\left(\mathrm{cm}^{3}\right)$} & \multicolumn{2}{|c|}{ GTV diameter (cm) } & \multicolumn{2}{|c|}{ GTV volume $\left(\mathrm{cm}^{3}\right)$} & \multicolumn{2}{|c|}{ Pulmonary function } \\
\hline & & & & $A$ & B & $A$ & B & $A$ & B & A & B & $A$ & $\mathrm{~B}$ \\
\hline 2 & M & 50 Gy/5 f & $\mathrm{P}$ & 62 & 69 & 3,819 & $2,754.7$ & 2.5 & 1.4 & 8 & 1.3 & 0 & 1 \\
\hline 5 & M & $60 \mathrm{~Gy} / 8 \mathrm{f}$ & $\mathrm{C}$ & 73 & 68 & 3,313 & $4,811.6$ & 3.4 & 5.4 & 20.7 & 81.2 & 0 & 0 \\
\hline 6 & M & $60 \mathrm{~Gy} / 8 \mathrm{f}$ & $\mathrm{C}$ & 57 & 66 & 2,614 & $4,777.6$ & 1.6 & 4 & 2.1 & 33.7 & 0 & 0 \\
\hline
\end{tabular}

F, female; M, male; f, fractions; C, central tumors: tumors are within or touching the zone of the proximal bronchial tree including those which are immediately adjacent to mediastinal or pericardial pleura (PTV touching the pleura). P, peripheral tumors: tumors are under the level three bronchus but above the respiratory bronchioles located around the lung. A, TKI plus SBRT group; B, SBRT alone group; BED, biologically effective dose; 0 , normal pulmonary function; 1 , slight pulmonary function injury; 2 , moderate pulmonary injury.

with gefitinib and concurrent docetaxel and radiation (18). Inoue $e t a l$. concluded that gefitinib caused acute interstitial pneumonitis due to the alveolar damages from radiation, and, thus, induced severer pulmonary toxic effects with the combined therapy than the radiation alone (22). The study showed that erlotinib can cause pulmonary interstitial injury and increase the risk of radiological pneumonitis (RP) $(23,24)$. Other studies of small sample sizes revealed that erlotinib combined with chest SBRT had a higher risk of $\mathrm{RP}(5,25)$. The incidence of interstitial lung disease caused by EGFR-TKIs or radiation may be low but fatal. A single institutional study demonstrated that 2 of 25 patients with advanced lung cancer treated with gefitinib/erlotinib followed by concomitant radiotherapy, died of radiationinduced lung injury at 3 months after radiotherapy (19).

One of the highlighted approaches of this study in quantitative CT imaging analysis. It should be noted that using CT number change to measure lung injury may not have a direct connection to patient's wellbeing. Because the lung volume receiving high radiation dose is limited in SBRT, the clinical symptom-based assessment does not necessarily evaluate the asymptomatic regional injury (26). Even though the radiographic changes in lung were obvious, SBRTinduced clinical pneumonitis was uncommon because of the small irradiated volume. However, as shown in Figure 2A, the dose response curve of $\mathrm{HU}$ changes clearly indicated that EGFR-TKI increased the radiation sensitivity of radiation lung damage. On the other hand, using CT intensity changes, as an endpoint to create a normal tissue probability model has advantage for dose response relationship, though not clinically most important to the patient. CT number changes can serve as subclinical endpoint, as it is the base of clinical symptom and, is often considered as surrogate endpoints. In this study, we elected the threshold of CT change at $200 \mathrm{HU}$, however, this number was elected in a relative arbitrary fashion. Diot et al. (14) suggested the density increase of $80 \mathrm{HU}$ may be a threshold of Radiation Therapy Oncology Group grades between 1 and 2. Our value was close to the dichotomous toxicity endpoint for lung density increase and was determined as a relevant parameter to complication probability in a previous study (13). The results from our study are exploratory and hypothesis generating. Further study with larger number of cases and longer followup is needed.

It should be noted that the results of this study have limitations. Firstly, it is noted that there are significant differences in patients' characteristics between the TKISBRT group and the SBRT alone group largely due to the nature of disease stage. Most of patients in the TKI-SBRT group were stage III/IV adenocarcinomas and non-smokers, while majority of patients treated with SBRT alone were male, smokers, and stage I/II disease. While perfectly matched cases are not possible, the visual inspection of baseline lung function mapping of the limited cases did provide us more knowledge. More quantitative analysis is needed in future studies. Secondly, Defraene et al. (27) reported the location of tumor could influence lung damage quantification on CT. But our study did not find significant differences of tumor location between two groups $(\mathrm{P}=0.276)$. Our research only included patients with small size of tumor treated with SBRT. The 
lower or upper lobe may not be an independent prognostic factor of density change. Most of the tumors location from the included patients were located in the upper or middle lobe. We got insufficient statistical power to analyze the effect of lower lobe on tumor density due to the limited sample size. Thirdly, our sample number is limited by the strict dose prescription and nature of retrospective selection of control cases. Although we had 242 patients with SBRT treatment of the lung available, only 20 primary NSCLC patients were treated with SBRT alone using the pre-defined dose regimen for primary NSCLC. This study is hypothesis generating. More rigorous studies with larger sample sizes and homogenous patient population are needed.

Despite these limitations, our study serves to propose a new dimension for interpreting clinical phenomena. The results suggest an increased risk of toxicity when treated with combined EGFR-TKI and SBRT, where unnoticed changes can be quantitatively analyzed.

\section{Conclusions}

The findings of our study demonstrated that prior EGFRTKI treatment may increase SBRT lung damage in NCSLC. This is likely the first study performed such a detailed quantitative imaging analysis with interesting hypothesis generating results in EGFR mutated patients. Further study with larger number of cases and long-term follow-up are warranted to further evaluate and validate our findings.

\section{Acknowledgments}

The authors thank all the physicians, physicist and radiographers who have been involved in the development of the stereotactic body radiotherapy program. The abstract of the study has been presented at the 2019 World Conference on Lung Cancer Meeting (September 7-9, Spain).

Funding: This work was supported in part by the Chinese National Science Foundation Projects (NSFC 81874221 and 81872458), Shenzhen Science and Technology Program (Grant No. KQTD20180411185028798), Zhejiang Provincial Medicine and Health Foundation (2015KYA240), Science and Technology Agency of Taizhou City (15yw01), China.

\section{Footnote}

Reporting Checklist: The authors have completed the STROBE reporting checklist. Available at http://dx.doi. org/10.21037/apm-20-1116

Data Sharing Statement: Available at http://dx.doi. org/10.21037/apm-20-1116

Peer Review File: Available at http://dx.doi.org/10.21037/ apm-20-1116

Conflicts of Interest: All authors have completed the ICMJE uniform disclosure form (available at http://dx.doi. org/10.21037/apm-20-1116). The authors have no other conflicts of interest to declare.

Ethical Statement: The authors are accountable for all aspects of the work in ensuring that questions related to the accuracy or integrity of any part of the work are appropriately investigated and resolved. The study was conducted in accordance with the Declaration of Helsinki (as revised in 2013). The study was approved by the Taizhou Hospital Ethical Committee (No. KB20170501) and informed consent was taken from all the patients.

Open Access Statement: This is an Open Access article distributed in accordance with the Creative Commons Attribution-NonCommercial-NoDerivs 4.0 International License (CC BY-NC-ND 4.0), which permits the noncommercial replication and distribution of the article with the strict proviso that no changes or edits are made and the original work is properly cited (including links to both the formal publication through the relevant DOI and the license). See: https://creativecommons.org/licenses/by-nc-nd/4.0/.

\section{References}

1. Tan DS, Yom SS, Tsao MS, et al. The International Association for the Study of Lung Cancer Consensus Statement on Optimizing Management of EGFR Mutation-Positive Non-Small Cell Lung Cancer: Status in 2016. J Thorac Oncol 2016;11:946-63.

2. Lilenbaum R, Samuels M, Wang X, et al. A phase II study of induction chemotherapy followed by thoracic radiotherapy and erlotinib in poor-risk stage III non-small-cell lung cancer: results of CALGB 30605 (Alliance)/RTOG 0972 (NRG). J Thorac Oncol 2015;10:143-7.

3. Swaminath A, Wright JR, Tsakiridis TK, et al. A Phase II Trial of Erlotinib and Concurrent Palliative Thoracic Radiation for Patients With Non-Small-Cell Lung Cancer. Clin Lung Cancer 2016;17:142-9. 
4. Iwama E, Nakanishi Y, Okamoto I, et al. Combined therapy with epidermal growth factor receptor tyrosine kinase inhibitors for non-small cell lung cancer. Expert Rev Anticancer Ther 2018;18:267-76.

5. Borghetti P, Bonu ML, Roca E, et al. Radiotherapy and tyrosine kinase inhibitors in stage iv non-small cell lung cancer: real-life experience. In Vivo 2018;32:159-64.

6. Kriegs M, Gurtner K, Can Y, et al. Radiosensitization of NSCLC cells by EGFR inhibition is the result of an enhanced p53-dependent G1 arrest. Radiother Oncol 2015;115:120-7.

7. Vanpouille-Box C, Diamond JM, Pilones KA, et al. TGFbeta Is a Master Regulator of Radiation TherapyInduced Antitumor Immunity. Cancer Res 2015; 75:2232-42.

8. Lv D, Wang W, Ding J, et al. Early SBRT to the primary tumor may overcome the Icotinib resistance in patients with advanced NSCLC harboring EGFR mutations. J Thorac Oncol 2018;13:S893.

9. Kataoka Y, Hirano K, Narabayashi T, et al. Concordance between the response evaluation criteria in solid tumors version 1.1 and the immune-related response criteria in patients with non-small cell lung cancer treated with nivolumab: a multicenter retrospective cohort study. Cancer Chemother Pharmacol 2018;81:333-7.

10. Kong FM, Hayman JA, Griffith KA, et al. Final toxicity results of a radiation-dose escalation study in patients with non-small-cell lung cancer (NSCLC): predictors for radiation pneumonitis and fibrosis. Int J Radiat Oncol Biol Phys 2006;65:1075-86.

11. Paul J, Yang C, Wu H, et al. Early Assessment of Treatment Responses During Radiation Therapy for Lung Cancer Using Quantitative Analysis of Daily Computed Tomography. Int J Radiat Oncol Biol Phys 2017; 98:463-72.

12. Orton CG, Cohen L. A unified approach to dose-effect relationships in radiotherapy. I: Modified TDF and linear quadratic equations. Int J Radiat Oncol Biol Phys 1988;14:549-56.

13. Diot Q, Kavanagh B, Stuhr K, et al. WE-G-BRA-07: Biological Modeling and Global Normal Lung Tissue Radiological Response in Patients Treated with Stereotactic Body Radiation Therapy for Lung Tumors. Med Phys 2011;38:3827.

14. Diot Q, Marks LB, Bentzen SM, et al. Comparison of radiation-induced normal lung tissue density changes for patients from multiple institutions receiving conventional or hypofractionated treatments. Int J Radiat Oncol Biol
Phys 2014;89:626-32.

15. Ren G, Ho WY, Qin J, et al. Deriving Lung Perfusion Directly from CT Image Using Deep Convolutional Neural Network: A Preliminary Study; Cham: Springer International Publishing, 2019.

16. Semenenko VA, Li XA. Lyman-Kutcher-Burman NTCP model parameters for radiation pneumonitis and xerostomia based on combined analysis of published clinical data. Phys Med Biol 2008;53:737-55.

17. Williams KJ, Telfer BA, Stratford IJ, et al. ZD1839 ('Iressa'), a specific oral epidermal growth factor receptortyrosine kinase inhibitor, potentiates radiotherapy in a human colorectal cancer xenograft model. Br J Cancer 2002;86:1157-61.

18. Center B, Petty WJ, Ayala D, et al. A phase I study of gefitinib with concurrent dose-escalated weekly docetaxel and conformal three-dimensional thoracic radiation followed by consolidative docetaxel and maintenance gefitinib for patients with stage III non-small cell lung cancer. J Thorac Oncol 2010;5:69-74.

19. Chang CC, Chi KH, Kao SJ, et al. Upfront gefitinib/ erlotinib treatment followed by concomitant radiotherapy for advanced lung cancer: a mono-institutional experience. Lung Cancer 2011;73:189-94.

20. Ramella S, Alberti AM, Cammilluzzi E, et al. Erlotinib and Concurrent Chemoradiation in Pretreated NSCLC Patients: Radiobiological Basis and Clinical Results. BioMed Res Int 2013;2013:403869.

21. Kroeze SG, Fritz C, Hoyer M, et al. Toxicity of concurrent stereotactic radiotherapy and targeted therapy or immunotherapy: A systematic review. Cancer Treat Rev 2017;53:25-37.

22. Inoue A, Saijo Y, Maemondo M, et al. Severe acute interstitial pneumonia and gefitinib. Lancet 2003; 361:137-9.

23. Ren S, Li Y, Li W, et al. Fatal Asymmetric Interstitial Lung Disease after Erlotinib for Lung Cancer. Respiration 2012;84:431-5.

24. Tsubata Y, Hamada A, Sutani A, et al. Erlotinib-induced acute interstitial lung disease associated with extreme elevation of the plasma concentration in an elderly non-small-cell lung cancer patient. J Cancer Res Ther 2012;8:154-6.

25. Nanda A, Dias-Santagata DC, Stubbs H, et al. Unusual tumor response and toxicity from radiation and concurrent erlotinib for non-small-cell lung cancer. Clin Lung Cancer 2008;9:285-7.

26. Stanic S, Paulus R, Timmerman RD, et al. No clinically 
significant changes in pulmonary function following stereotactic body radiation therapy for early- stage peripheral non-small cell lung cancer: an analysis of RTOG 0236. Int J Radiat Oncol Biol Phys 2014; 88:1092-9.

Cite this article as: Tang $\mathrm{X}$, Shen $\mathrm{Y}$, Meng $\mathrm{Y}$, Hou L, Zhou C, Yu C, Jia H, Wang W, Ren G, Cai J, Li XA, Yang H, Kong FM. Radiation-induced lung damage in patients treated with stereotactic body radiotherapy after EGFR-TKIs: is there any difference from stereotactic body radiotherapy alone? Ann Palliat Med 2021;10(3):2832-2842. doi: 10.21037/apm-20-1116
27. Defraene G, Van Elmpt W, Crijns W, et al. Regional variability in radiation-induced lung damage can be predicted by baseline CT numbers. Radiother Oncol 2017;122:300-6. 